\title{
Handling binary classification problems with a priority class by using support vector machines \\ L. Gonzalez-Abril ${ }^{\mathrm{a}}$, C. Angulo ${ }^{\mathrm{b}}, \mathrm{H} . \mathrm{Nuñez}^{\mathrm{c}}, \mathrm{Y}_{\text {. Leal }}^{\mathrm{d}}$ \\ ${ }^{a}$ Dpto. Economía Aplicada I, Universidad de Sevilla, 41018 Sevilla, Spain \\ ${ }^{b}$ Automatic Control Dept. - Univ. Politècnica de Catalunya, 08028 Barcelona - Spain \\ ${ }^{c}$ AI Lab. Facultad de Ciencias, Univ. Central Venezuela, 1020-A Caracas, Venezuela \\ ${ }^{d}$ Unitat of Diabetes, Endocrinology and Nutrition (UDEN), Institut d'Investigació Biomédica de Girona (IdIBGi), 17007 Girona, Spain
}

\begin{abstract}
A post-processing technique for Support Vector Machine (SVM) algorithms for binary classification problems is introduced in order to obtain adequate accuracy on a priority class (labelled as a positive class). That is, the true positive rate (or recall or sensitivity) is prioritized over the accuracy of the overall classifier. Hence, false negative (or Type I) errors receive greater consideration than false positive (Type II) errors during the construction of the model.

This post-processing technique tunes the initial bias term once a solution vector is learned by using standard SVM algorithms in two steps: First, a fixed threshold is given as a lower bound for the recall measure; second, the true negative rate (or specificity) is maximized.

Experiments, carried out on eleven standard UCI datasets, show that the modified SVM satisfies the aims for which it has been designed. Furthermore, results are comparable or better than those obtained when other state-of-theart SVM algorithms and other usual metrics are considered.
\end{abstract}

Key words: support vector machines, post-processing strategies, pattern recognition, cost-sensitive SVM

\section{Introduction}

There exist situations where the correct detection of instances of one class (the positive class) is considered to be of greater importance or priority than 
the other class (the negative class) in binary classification problems. Problems which involve this situation arise: in the medical diagnosis of certain disorders, such as the detection of breast cancer [16, 30] or cardiac care [23]; in certain financial problems, such as credit-card fraud detection [29], financial crisis [18], detection of financial statement fraud [24, 6], bankruptcy prediction [27, 21], prediction of liquefaction potential [28] and bank marketing [20]; and in criminological investigations, among other applications.

For these kinds of problems, the attainment of a high percentage for the true positive rate (also named recall or sensitivity) is more important than the overall accuracy measure. That is, Type I errors should receive serious consideration during the model construction. For these cases, models should therefore exhibit a high performance in the priority class, while simultaneously striving to maintain a low error performance in the non-priority class. For instance, when classifying whether a firm is bankrupt, Type I error occurs when the classifier incorrectly classifies a bankrupt firm into the non-bankrupt class. Type II errors are based on the classifier incorrectly classifying a non-bankrupt firm into the bankrupt class. A higher Type I error rate incurs greater costs on financial institutions, which can enhance business risk.

In order to handle this kind of problem, the application of Support Vector Machines (SVMs) is a popular choice in the machine-learning research area since these are learning machines that implement the structural-riskminimization inductive principle to obtain good generalization on a limited number of learning patterns $[25,26,22]$. The theory of SVMs was developed on the basis of a separable binary classification problem where the optimization criterion is the width of the margin with $\ell_{2}$-norm ${ }^{1}$, between the positive and negative examples. An SVM with a large margin separating two classes has a small Vapnik-Chervonenkis dimension, which provides good generalization performance [5].

Furthermore, SVMs present an attractive option for binary problems with a priority class, since they can be modified in order to incorporate information on the penalties associated with erroneous predictions for each class into the learning problem . Therefore, SVMs can afford to prioritize one class over the other.

The main contribution in this paper is that once the solution vector for

\footnotetext{
${ }^{1} \mathrm{~A}$ generalization is given in [13].
} 
Table 1: The confusion matrix for a classifier $f$ on a test set $\mathcal{D}$.

\begin{tabular}{c|c|c}
\hline Actual & Positive & Negative \\
\hline Predicted & True Positive $\left(t_{\text {pos }}\right)$ & False Positive $\left(f_{\text {pos }}\right)$ \\
\hline Positive & False Negative $\left(f_{\text {neg }}\right)$ & True Negative $\left(t_{\text {neg }}\right)$ \\
\hline Number of instances & $N_{\text {pos }}$ & $N_{\text {neg }}$
\end{tabular}

the standard SVM problem is obtained, the bias is considered as a parameter to be tuned in order to improve the generalization performance on the non-priority class, whereas a generalization performance with a threshold is maintained on the priority class.

It must be emphasized that the proposed solution neither modifies the original optimization problem for SVM training, nor introduces new hyperparameters. Thus, no increase in the computational cost is incurred.

The remainder of this paper is organized as follows: Section 2 introduces the metrics commonly employed in classification problems. In Section 3, the standard SVM approach is outlined and a family of classifiers that depends on the bias is considered. Furthermore, certain results on the metrics are obtained. An SVM, called Biased SVM (BSVM), is introduced in Section 4, based on both recall and specificity metrics. Experiments are carried out in Section 5 which illustrate the performance of the BSVM in comparison with the standard SVM and the well-known cost-sensitive SVM approach. Finally, conclusions are drawn.

\section{Metrics}

Let $\mathcal{Z}=\left\{\left(x_{1}, y_{1}\right), \ldots,\left(x_{N}, y_{N}\right)\right\}$ be a training set, with $x_{i} \in \mathcal{X} \subset \mathbb{R}^{d}$, $y_{i} \in \mathcal{Y}=\{+1,-1\}$, and $z_{i}=\left(x_{i}, y_{i}\right)$. Let $f(x)$ be a binary classifier such that outputs are obtained as $h(x)=\operatorname{sign}(f(x))$, where $\operatorname{sign}(\cdot)$ is the sign function. Let $\mathcal{Z}_{\text {pos }}=\left\{z_{i} \in \mathcal{Z} \mid y_{i}=+1\right\} \neq \emptyset$ and $\mathcal{Z}_{\text {neg }}=\left\{z_{i} \in \mathcal{Z} \mid y_{i}=-1\right\} \neq \emptyset$ be the sets of training patterns for the positive class and the negative class, respectively. Let $N_{\text {pos }}=\# \mathcal{Z}_{\text {pos }}$ and $N_{\text {neg }}=\# \mathcal{Z}_{\text {neg }}$ be the number of positive and negative instances, respectively. Hence $N=N_{\text {pos }}+N_{\text {neg }}$.

Based on the confusion matrix or contingency table (see Table 1), the most commonly used metric for the evaluation of the generalization perfor- 
mance of the classifier $f(x)$ on a test set $\mathcal{D} \subset \mathcal{X} \times \mathcal{Y}$ is the accuracy, denoted by $A c$, which computes the proportion of instances that are correctly classified by the model, that is,

$$
A c(f, \mathcal{D})=\frac{t_{\text {pos }}+t_{\text {neg }}}{N_{\text {pos }}+N_{\text {neg }}}
$$

Using this metric, both classes, positive and negative, have the same priority for the purpose of classification. Hence, accuracy can be deceiving when:

1. one class is considered of greater significance than the other class. Thus, the cost for wrong classifications in the priority class (Type I error) is greater than the cost for wrong classifications in the other class (Type II error). For instance, a false negatives, which may deny medical treatment to a patient, is more critical than a false positive that leads to carrying out a medical check on a healthy person; and

2. prior probabilities of classes differ greatly (imbalanced datasets), since the metrics fail to consider costs for wrong classifications, and thus remain very sensitive to the bias between classes [14]. For instance, when detecting fraud in credit-card transactions, the ratio between fraudulent and healthy transaction instances is around 1/6000; a naive approach of classifying every example to be a negative instance would provide a high accuracy, however this description fails to reflect the fact that none of the fraudulent transactions is detected.

Therefore, other measures of assessment must be considered. By considering accuracy rates on $\mathcal{Z}_{\text {pos }}$ and $\mathcal{Z}_{\text {neg }}$ separately, the recall ${ }^{2}$ and specificity metrics, denoted by $R e$ and $S p$, respectively, are defined as follows:

$$
R e(f, \mathcal{D})=\frac{t_{\text {pos }}}{N_{\text {pos }}}, \quad \text { and } \quad S p(f, \mathcal{D})=\frac{t_{\text {neg }}}{N_{\text {neg }}} .
$$

The recall measure is the proportion of positive cases that are correctly identified (the true positive rate), that is, one minus the proportion of Type I error. On the other hand, specificity is the fraction of correctly identified examples among all instances that are negative (the true negative rate), that

\footnotetext{
${ }^{2}$ Also called sensitivity in certain fields.
} 
is, one minus the proportion of Type II error. Both metrics are measures of completeness.

Hence, a new expression for accuracy can be derived as follows:

$$
A c(f, \mathcal{D})=\frac{N_{\text {pos }} \cdot R e(f, \mathcal{D})+N_{\text {neg }} \cdot S p(f, \mathcal{D})}{N_{\text {pos }}+N_{\text {neg }}}
$$

as a weighted arithmetic mean of recall and specificity, where the associated weights are the number of positive instances and negative instances, respectively.

In the case that the set $\mathcal{Z}_{\text {pos }}$ is considered a "priority" for the purpose of classification, then the $\operatorname{Re}(f, \mathcal{D})$ measure is more representative than the $S p(f, \mathcal{D})$ measure, and $A c(f, \mathcal{D})$ is no longer an adequate metric [8].

Therefore, a new approach to obtain a classifier when one class is considered a "priority" is introduced: For a fixed test set $\mathcal{D} \subset \mathcal{X} \times \mathcal{Y}$, when seeking for a good classifier, the search is restricted to classifiers that hold recall measures at a specified level. Within this set of classifiers, a classifier is sought in order to maximize specificity. It is worth noting that, in general, it is not possible to arbitrarily obtain great recall and specificity values, that is, the capacity of any classifier cannot increase the number of the true positives without also increasing the number of false positives.

According to the proposed approach, the following well-known metrics will be also considered in the measurement of the performance of the classifiers:

- The geometric mean (g-mean), defined as

$$
G_{\text {mean }}(f, \mathcal{D})=\sqrt{\operatorname{Re}(f, \mathcal{D}) \cdot S p(f, \mathcal{D})} .
$$

- The precision, given by

$$
\operatorname{Pr}(f, \mathcal{D})=\frac{t_{\text {pos }}}{t_{\text {pos }}+f_{\text {pos }}} .
$$

- The $F_{\text {value }}$ metric (f-value), defined as

$$
F_{\text {value }}(f, \mathcal{D})=\left(1+\beta^{2}\right) \cdot \frac{\operatorname{Pr}(f, \mathcal{D}) \cdot \operatorname{Re}(f, \mathcal{D})}{\beta^{2} \operatorname{Re}(f, \mathcal{D})+\operatorname{Pr}(f, \mathcal{D})},
$$

where $\beta$ is a tuning parameter (usually, $\beta=1$ ). 


\section{Support Vector Machine and Bias}

Let $\phi: \mathcal{X} \rightarrow \mathcal{H}, \mathrm{x}=\phi(x)$ be a mapping to a feature space endowed with a dot product denoted by $\langle\cdot, \cdot\rangle$. A binary linear classifier $f: \mathcal{X} \rightarrow \mathbb{R}$, defined as $^{3} f(x)=\langle\mathrm{x}, \mathrm{w}\rangle-b$, is sought, where $\mathrm{w} \in \mathcal{H}, b \in \mathbb{R}$. Outputs are obtained as $h(x)=\operatorname{sign}(f(x))$.

\subsection{The standard primal SVM}

The standard SVM formulation leads to the optimization problem [10]:

$$
\begin{aligned}
& \min _{\mathrm{w} \in \mathcal{H}, b \in \mathbb{R}} \frac{1}{2}\|\mathrm{w}\|^{2}+C \sum_{i=1}^{N} \xi_{i} \\
& \text { s.t. }\left\{\begin{array}{l}
y_{i}\left(\left\langle\mathrm{x}_{\mathrm{i}}, \mathrm{w}\right\rangle-b\right)+\xi_{i} \geq 1, \\
\xi_{i} \geq 0, \quad z_{i} \in \mathcal{Z} \quad i=1, \cdots, N
\end{array}\right.
\end{aligned}
$$

where $\xi_{i}$ are slack variables and $C>0$ acts as a term of regularization.

The solution of this problem can be written as $\mathrm{w}_{0}=\sum_{i} \gamma_{i} y_{i} \mathrm{x}_{\mathrm{i}}$, where $\mathrm{x}_{\mathrm{i}}=\phi\left(x_{i}\right)$ and $\gamma_{i}$ are Lagrange multipliers for the dual formulation of (3), with $\sum_{i} \gamma_{i} y_{i}=0$. Term $b$ is calculated a posteriori [11], and is denoted by $b_{0}$ (bias standard). Hence, the classifier can be written as

$$
f(x)=\sum_{i=1}^{N} \gamma_{i} y_{i} K\left(x_{i}, x\right)-b_{0}=\left\langle\mathrm{x}, \mathrm{w}_{0}\right\rangle-b_{0}
$$

where $K: \mathcal{X} \times \mathcal{X} \rightarrow \mathbb{R}$, defined as $K(x, y)=\langle\mathrm{x}, \mathrm{y}\rangle=\langle\phi(x), \phi(y)\rangle$, is called the kernel function.

\subsection{The cost-sensitive SVM}

The cost-sensitive SVM modifies the standard optimization problem (3) in order to incorporate information on the penalties associated with erroneous predictions for each class into the learning problem [7]. Thus, the two types of errors can be introduced into the formulation of the learning problem, using two regularization parameters, as follows [4]:

$$
\begin{aligned}
& \min _{\mathbf{w} \in \mathcal{H}, b \in \mathbb{R}} \frac{1}{2}\|\mathbf{w}\|^{2}+C^{+} \sum_{i \mid y_{i}=+1} \xi_{i}+C^{-} \sum_{i \mid y_{i}=-1} \xi_{i} \\
& \text { s.t. }\left\{\begin{array}{l}
y_{i}\left(\left\langle\mathrm{x}_{\mathrm{i}}, \mathrm{w}\right\rangle-b\right)+\xi_{i} \geq 1, \\
\xi_{i} \geq 0, \quad z_{i} \in \mathcal{Z} \quad i=1, \cdots, N
\end{array}\right.
\end{aligned}
$$

\footnotetext{
${ }^{3}$ For notation consistency, bias $b$ is incorporated with a negative sign.
} 
where $C^{+}$and $C^{-}$are the costs associated with errors in the positive class and negative class, respectively.

Furthermore, the solution vector can be written in the same way as the solution vector of the optimization problem (3).

It is worth noting that the cost-sensitive SVM usually provides a better recall measure than does the standard SVM. Nevertheless, this approach fails to provide a way to regulate the level of recall. The SVM proposed in this paper regulates via the bias tuning.

\subsection{The recall and specificity metrics}

A key point of the proposed approach is that once the solution vector $\mathrm{w}_{0}$ is obtained using the standard SVM formulation, the bias $b$ can be considered as a parameter. In accordance with to the notation in [12], the set of classifiers $\mathcal{F}\left(\mathrm{w}_{0}\right)$ defined as

$$
\left\{f_{b}: \mathcal{X} \rightarrow \mathbb{R}, f_{b}(\cdot)=\left\langle\phi(\cdot), \mathrm{w}_{0}\right\rangle-b, b \in \mathbb{R}\right\}
$$

is considered. A map $\Theta_{b}: \mathcal{X} \rightarrow\{-1,+1\}$ is also defined, and is associated to the classifier $f_{b}(x) \in \mathcal{F}\left(\mathrm{w}_{0}\right)$ such that, given an input vector $x$, it assigns a label as follows:

$$
\Theta_{b}(x)=\operatorname{sign}\left(f_{b}(x)\right)= \begin{cases}+1 & \text { if }\left\langle\mathrm{x}, \mathrm{w}_{0}\right\rangle \geq b \\ -1 & \text { if }\left\langle\mathrm{x}, \mathrm{w}_{0}\right\rangle<b .\end{cases}
$$

Let us define the recall function, $R e: \mathbb{R} \rightarrow[0,1]$, as $\operatorname{Re}(b)=\operatorname{Re}\left(f_{b}, \mathcal{Z}\right)$ and the specificity function, $S p: \mathbb{R} \rightarrow[0,1]$, as $S p(b)=S p\left(f_{b}, \mathcal{Z}\right)$ from (1), where $f_{b} \in \mathcal{F}\left(\mathrm{w}_{0}\right)$.

Furthermore, let order

$$
\mathcal{Z}_{\text {pos }}=\left\{\left(\mathrm{x}_{1},+1\right), \cdots,\left(\mathrm{x}_{N_{\text {pos }}},+1\right)\right\}=\left\{\left(\mathrm{p}_{1},+1\right), \cdots,\left(\mathrm{p}_{N_{\text {pos }}},+1\right)\right\}
$$

with $\mathrm{p}_{i}=\mathrm{x}_{\sigma^{*}(i)}$, where $\sigma^{*}$ is a permutation of $N_{\text {pos }}$ such that

$$
\left\langle\mathrm{p}_{1}, \mathrm{w}_{0}\right\rangle \leq \cdots \leq\left\langle\mathrm{p}_{\mathrm{i}}, \mathrm{w}_{0}\right\rangle \leq \cdots \leq\left\langle\mathrm{p}_{N_{p o s}}, \mathrm{w}_{0}\right\rangle
$$

for $i=1, \cdots, N_{\text {pos }}$.

Let us consider the values

$$
\beta=\min _{z_{i} \in \mathcal{Z}_{\text {pos }}}\left\langle\mathrm{x}_{\mathrm{i}}, \mathrm{w}_{0}\right\rangle=\left\langle\mathrm{p}_{1}, \mathrm{w}_{0}\right\rangle \quad \text { and } \quad \beta^{*}=\max _{z_{i} \in \mathcal{Z}_{\text {pos }}}\left\langle\mathrm{x}_{\mathrm{i}}, \mathrm{w}_{0}\right\rangle=\left\langle\mathrm{p}_{N_{\text {pos }}}, \mathrm{w}_{0}\right\rangle .
$$

Hence, by defining the bias $b_{i}=\left\langle\mathrm{p}_{\mathrm{i}}, \mathrm{w}_{0}\right\rangle$ for $i=1, \cdots, N_{\text {pos }}$, the following results hold: 
- $\operatorname{Re}(b)$ is a decreasing function of $b$.

- $\operatorname{Re}\left(b_{i}\right) \geq \frac{N_{\text {pos }}-(i-1)}{N_{\text {pos }}} \forall i$. Furthermore, if $b_{i} \neq b_{j}$ for $i \neq j$, then $\operatorname{Re}\left(b_{i}\right)=$ $\frac{N_{\text {pos }}-(i-1)}{N_{\text {pos }}}$ and $\operatorname{Re}\left(b_{i}\right)=\operatorname{Re}\left(b_{i+1}\right)+\frac{1}{N_{\text {pos }}}$.

- If $b_{i}<b_{i+1}$, then $\operatorname{Re}\left(b_{i}\right)>\operatorname{Re}(b)=\operatorname{Re}\left(b_{i+1}\right)$ for any $b$ such that $b_{i}<b \leq b_{i+1}$.

- If $b \leq \beta<b^{\prime} \leq \beta^{*}<b^{*}$, then $\operatorname{Re}(b)=1>\operatorname{Re}\left(b^{\prime}\right)>0=\operatorname{Re}\left(b^{*}\right)$.

Analogously, let

$$
\mathcal{Z}_{\text {neg }}=\left\{\left(\mathrm{x}_{1},-1\right), \cdots,,\left(\mathrm{x}_{N_{\text {neg }}},-1\right)\right\}=\left\{\left(\mathrm{q}_{1},-1\right), \cdots,,\left(\mathrm{q}_{N_{\text {pos }}},-1\right)\right\}
$$

with $\mathrm{q}_{j}=\mathrm{x}_{\sigma^{\prime}(j)}$ where $\sigma^{\prime}$ is a permutation of $N_{\text {neg }}$ such that

$$
\left\langle\mathrm{q}_{1}, \mathrm{w}_{0}\right\rangle \leq \cdots \leq\left\langle\mathrm{q}_{\mathrm{j}}, \mathrm{w}_{0}\right\rangle \leq \cdots \leq\left\langle\mathrm{q}_{N_{n e g}}, \mathrm{w}_{0}\right\rangle
$$

for $j=1, \cdots, N_{\text {neg }}$.

Let us consider the values

$\alpha^{*}=\min _{z_{i} \in \mathcal{Z}_{\text {neg }}}\left\langle\mathrm{x}_{\mathrm{i}}, \mathrm{w}_{0}\right\rangle=\left\langle\mathrm{q}_{1}, \mathrm{w}_{0}\right\rangle \quad$ and $\quad \alpha=\max _{z_{i} \in \mathcal{Z}_{\text {neg }}}\left\langle\mathrm{x}_{\mathrm{i}}, \mathrm{w}_{0}\right\rangle=\left\langle\mathrm{q}_{N_{\text {neg }}}, \mathrm{w}_{0}\right\rangle$.

Hence, by considering the bias $b q_{j}=\left\langle\mathrm{q}_{\mathrm{j}}, \mathrm{w}_{0}\right\rangle$ for $j=1, \cdots, N_{\text {neg }}$, it follows that:

- $S p(b)$ is an increasing function of $b$.

- $S p\left(b q_{j}\right) \leq \frac{j-1}{N_{\text {neg }}}$. Furthermore, if $b q_{i} \neq b q_{j}$ for $i \neq j$, then $S p\left(b q_{j}\right)=\frac{j-1}{N_{\text {neg }}}$ and $S p\left(b q_{j}\right)+\frac{1}{N_{\text {neg }}}=S p\left(b q_{j+1}\right)$.

- If $b q_{j}<b q_{j+1}$, then $S p\left(b q_{j}\right)<S p(b) \leq S p\left(b q_{j+1}\right)$ for any $b$ such that $b q_{j}<b \leq b q_{j+1}$.

- If $b \leq \alpha^{*}<b^{\prime} \leq \alpha<b^{*}$, then $S p(b)=0<S p\left(b^{\prime}\right)<1=S p\left(b^{*}\right)$.

By applying a toy dataset, an example for both metrics is depicted in Figure 1 , where $\mathrm{x}=\left(x_{1}, x_{2}\right), \mathrm{w}_{0}=(1,0)^{t}$, and $f_{b}(\mathrm{x})=x_{1}-b$. It can be verified that $N_{\text {pos }}=4, N_{\text {neg }}=5, \alpha=2, \alpha^{*}=-3, \beta=-2$, and $\beta^{*}=3$.

As expected, recall and specificity are inverse measures in the sense that an improvement of one of them implies a deterioration of the other. 

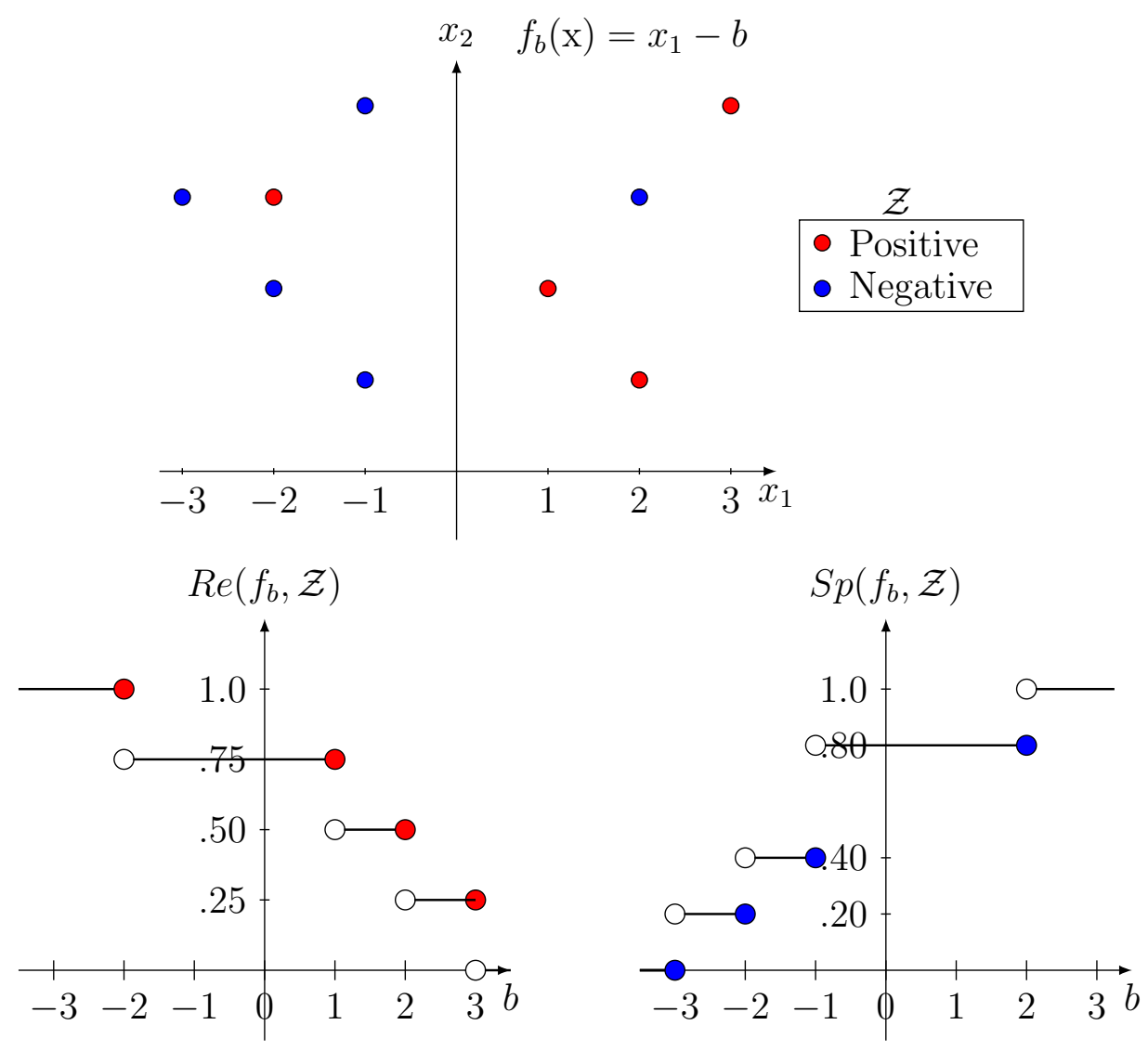

Figure 1: Graphical representation on a toy dataset of the recall and the specificity metrics with bias $b$ taken as a parameter.

\section{BSVM: Biased Support Vector Machine}

Within the set of classifiers $\mathcal{F}\left(\mathrm{w}_{0}\right)$, a tradeoff must be found between $R e(b)$, a decreasing function of $b$, and $S p(b)$, an increasing function of $b$, in order to maximize generalization.

By assuming that the positive class is of a higher priority than the negative class, then $\beta=\max \{b \in \mathbb{R}, \operatorname{Re}(b)=1\}$ is the best bias for the training set $\mathcal{Z}$, which leads to the classifier $f_{\beta}(x)=\left\langle\mathrm{x}, \mathrm{w}_{0}\right\rangle-\beta$. Nevertheless, $S p(\beta)$ can be arbitrarily small if the instance $\mathrm{p}_{1}$ is an outlier. It can be seen in Figure 1 that if $\beta=-2$, then $\operatorname{Re}(-2)=1, S p(-2)=0.20$ and $A c(-2)=\frac{5}{9}=0.5556$.

One way to circumvent this problem, given the set of classifiers $\mathcal{F}\left(\mathrm{w}_{0}\right)$, 
is to fix a threshold $0 \leq r \leq 1$, such that $R e(b) \geq r$ in order to guarantee a minimum true positive rate in the positive class and to maximize $S p(b)$. For example, from Figure 1, if $r=0.70$ then it can be seen that by considering $b=1$, one obtains $\operatorname{Re}(1)=0.75>0.70, S p(1)=0.80$, and $A c(1)=\frac{7}{9}=$ 0.7778 .

Hence, the following problem is considered:

$$
\begin{aligned}
& \max _{b \in \mathbb{R}} S p(b) \\
& \text { s.t. } \operatorname{Re}(b) \geq r, 0 \leq r \leq 1, f_{b} \in \mathcal{F}\left(\mathrm{w}_{0}\right)
\end{aligned}
$$

Proposition 4.1. A classifier $f_{b}(x) \in \mathcal{F}\left(\mathrm{w}_{0}\right)$ exists such that it is a solution of the problem (6).

\section{Proof:}

Let us consider the two possible cases:

- If $\alpha<\beta$, then $\mathcal{Z}$ is a linearly separable training set [9], and hence, for any $b$ such that $\alpha<b<\beta, \operatorname{Re}(b)=1$ and $S p(b)=1$ from the results given in Section 3, whereby both metrics obtain the maximum value. Therefore, $b$ is a solution of the problem (6) for any $0 \leq r \leq 1$.

In this case, the bias $b_{r}^{*}=(1-r) \beta+r \alpha$ is a solution of (6) such that $100 r \%$ of the space between $\alpha$ and $\beta$ is given for the positive instances. Hence, if $r$ is near to 1 , more space is given to the positive class (priority class) than to the negative class in order to carry out a better generalization with the positive class than with the negative class.

- If $\alpha \geq \beta$, then by taking into account that $R e\left(b_{i}\right) \geq \frac{N_{\text {pos }}-(i-1)}{N_{\text {pos }}}$ and by imposing $r \leq \frac{N_{\text {pos }}-(i-1)}{N_{\text {pos }}}$, it is obtained that $i \leq 1+N_{\text {pos }}(1-r)$. Hence, the $b_{i r}$ bias is considered, where $i r=\max \left\{i, i \leq 1+N_{\text {pos }}(1-r)\right\}$, that is, $b_{i r}$ is the (1-r)th q-quantile of $\mathcal{Z}_{\text {pos }}$.

Clearly, $b_{i r}$ exists since $\mathcal{Z}_{\text {pos }}$ is a non-empty and finite set. Furthermore, if $b>b_{i r}$, then $\operatorname{Re}(b) \leq R e\left(b_{i r+1}\right)=\frac{N_{\text {pos }}-(i r+1-1)}{N_{\text {pos }}}<r$. Therefore, since the specificity function is an increasing function, the $b_{i r}$ bias is a solution of the problem (6) for $0 \leq r \leq 1$.

The proof is completed. 
From the optimization problem (6), a mixed SVM is proposed, which is called Biased SVM (BSVM), where the solution of the problem (3) is tuned in order to obtain $R e\left(b_{r}\right) \geq r$, and specificity is maximized.

It should be indicated that the proposed approach can be viewed as hypothesis testing from Inference Statistics [3], that is, the value $(1-r)$ in the BSVM approach is similar to the significance level $\alpha$ in hypothesis testing.

Therefore, given an $r$ value such that $0 \leq r \leq 1$, then the classifier $f \in \mathcal{F}\left(\mathrm{w}_{0}\right)$ considered is as follows:

$$
f(x)=f_{b_{r}}(x)=\sum_{i=1}^{N} \gamma_{i} y_{i} K\left(x_{i}, x\right)-b_{r}=\left\langle\mathrm{x}, \mathrm{w}_{0}\right\rangle-b_{r}
$$

where

$$
b_{r}=\left\{\begin{array}{cc}
b_{r}^{*} & \text { if } \alpha<\beta \\
b_{i r} & \text { otherwise. }
\end{array}\right.
$$

Given a set of classifiers $\mathcal{F}\left(\mathrm{w}_{0}\right)=\left\{f_{b}(x)=\left\langle\mathrm{x}, \mathrm{w}_{0}\right\rangle-b, b \in \mathbb{R}\right\}$ on a training set $\mathcal{Z}$, the influence of the threshold on the performance of the BSVM can be analysed: for $0 \leq r \leq r^{\prime} \leq 1$, the $b_{r^{\prime}}$ bias is a solution of (6) for $r^{\prime}$ and verifies that $\operatorname{Re}\left(b_{r^{\prime}}\right) \geq r^{\prime} \geq r$, and therefore $S p\left(b_{r^{\prime}}\right) \leq S p\left(b_{r}\right)$, where $b_{r}$ is a solution of (6) for $r$. Furthermore, since $S p(b)$ is an increasing function of $b$, then $b_{r} \geq b_{r^{\prime}}$ and, since $R e(b)$ is a decreasing function of $b$, then $\operatorname{Re}\left(b_{r}\right) \leq \operatorname{Re}\left(b_{r^{\prime}}\right)$.

Therefore, if $0 \leq r \leq 1$, then $\operatorname{Re}\left(b_{r}\right)$ is an increasing function of $r$, and $S p\left(b_{r}\right)$ is a decreasing function of $r$ on the training set $\mathcal{Z}$. This result can be seen clearly in the experimentation given in the following section.

\section{Experimentation}

To the best our knowledge, there is no machine-learning approach for classification problems with an associated optimization problem similar to the problem proposed (6). Learning machines tend to strive to maximize the recall and specificity metrics simultaneously. No lower bound is determined for the recall measure on the training set. Hence, a comparative is carried out with the standard SVM and the cost-sensitive SVM in order to analyse the behaviour of the BSVM.

Experimentation is conducted on the following standard UCI datasets [2]: Iris plants, Teaching assistant evaluation, Glass identification database, Thyroid disease, protein localization sites (Ecoli), Bupa liver disorders, 
Table 2: UCI datasets used in the experimentation. The number in parentheses indicates the priority class. These datasets are ordered in terms of the number of instances.

\begin{tabular}{l|r|c|l} 
datasets & total & priority & brief description \\
\hline Iris (2) & 150 & 50 & The best known dataset for classification \\
Tae (1) & 151 & 49 & Evaluations of teaching performance \\
Glass (1) & 214 & 70 & Motivated by criminological investigation \\
Thyroid (3) & 215 & 30 & Thyroid disease \\
Ecoli (6) & 331 & 52 & Localization site of protein \\
Bupa (1) & 345 & 145 & Medical diagnosis of livers \\
Japanese (1) & 653 & 203 & Relative to financial problems \\
Australian (1) & 690 & 307 & Relative to financial problems \\
Pima (2) & 768 & 268 & Medical diagnosis of diabetes \\
German (2) & 1000 & 300 & Relative to financial problems \\
Bank (1) & 4521 & 521 & Relative to financial problems
\end{tabular}

Japanese credit screening, Australian credit approval, Pima Indians diabetes, German credit data, and Bank marketing ${ }^{4}$. A summary of the characteristics and a brief description of these data sets is shown in Table 2.

In order to apply the BSVM, datasets are split in the form of one class (the priority class), indicated in the first column, versus the rest of the classes. The priority class has been chosen with a lower number of examples than the non-priority class, that is, there is an imbalanced ratio between positive and negative instances $[17,19]$. This selection is considered in order to better illustrate the performance of the BSVM.

Three experiments are considered:

- In the first experiment, the performance of the BSVM with a lineal kernel is analyzed.

- Next, a similar approach is carried out with a lineal kernel where the priority is changed with respect to the former experiment in several datasets.

- In the last experiment, the rbf kernel is used instead of the linear kernel.

\footnotetext{
${ }^{4}$ For this dataset only the quantitative features have been considered.
} 
The first experiment is carried out by following a similar experimental framework to that used in [1] as suggested in [15]. Performance is evaluated on SVM classifiers using the linear kernel, which is chosen as a baseline for the empirical evaluation, and the regularization term $C$ is explored on a onedimensional grid with the following values: $C=\left[2^{-4}, 2^{-3}, \ldots, 2^{9}, 2^{10}\right]$. A cost-sensitive SVM, denoted by CS-SVM, is employed to train an SVM on the listed UCI datasets. The Matlab Bioinformatic toolbox is used where the values for $C^{+}$and $C^{-}$in (4) are calculated from $C$ as,

$$
C^{+}=\frac{C \cdot N}{2 N_{p o s}}, \quad C^{-}=\frac{C \cdot N}{2 N_{n e g}} .
$$

The criterion employed for the estimation of the generalized accuracy in the standard SVM and the cost-sensitive SVM, and the specificity in the BSVM is the three-fold $\left(N_{\text {fold }}=3\right)$ cross-validation on the whole set of training data. This procedure is repeated 100 times in order to ensure good statistical behaviour. The values considered for $r$, the minimum true positive rate in the positive class for the training set, are set to $0.85,0.90$, and 0.95 , following a similar experimental framework for hypothesis testing. It is worth noting that optimization problems (3), (4) and (6) are carried out on the training set, whereas results are given on the test set, hence recall values on the test set are not always greater or equal to the selected $r$ bound when training the BSVM.

The results obtained, given as percentages, are displayed in Table 3. Several conclusions can be drawn from this empirical experimentation:

- It is observed that specificity values are greater than recall values in the standard SVM (except for the Iris, Australian and German datasets). As mentioned earlier, the standard SVM is inherently biased towards the majority class when classifying imbalanced datasets. This is the main difference between SVM and BSVM, since BSVM focuses on the priority class regardless of whether it is the majority class.

- Due to the generalization capacity of BSVM, recall values on the test set are about $85 \%, 90 \%$ and $95 \%$ for all datasets, respectively. Hence, empirical results show that BSVM produces a good hypothesis in terms of recall, by using the optimization problem of the same SVM. It should be noted that recall value is greater (near to $95 \%$ of $r$ ) for the Thyroid dataset, since this optimization problem is linearly separable and the chosen bias is $b_{r}^{*}(7)$. 
Table 3: Average figures of the results of the experiment with a linear kernel.

\begin{tabular}{|c|c|c|c|c|c|c|c|c|}
\hline datasets & approach & $100 r$ & recall & pecificity & accuracy & g-mean & precision & f-value \\
\hline \multirow[t]{5}{*}{ Iris $(2)$} & SVM & *** & 79.35 & 66.07 & 70.35 & 72.41 & 53.90 & 64.20 \\
\hline & CS-SVM & $* * *$ & 68.21 & 81.49 & 72.64 & 74.14 & 88.51 & 76.63 \\
\hline & & 85 & 82.02 & 62.86 & 69.31 & 71.80 & 52.48 & 64.00 \\
\hline & BSVM & 90 & 86.91 & 57.20 & 67.16 & 70.51 & 50.38 & 63.78 \\
\hline & & 95 & 92.37 & 49.11 & 63.49 & 67.35 & 47.58 & 62.80 \\
\hline \multirow[t]{5}{*}{$\bar{T}$ Tae (1) } & $\overline{\text { SVM }}$ & *** & 55.14 & 76.77 & 68.85 & 65.06 & 53.28 & 54.19 \\
\hline & CS-SVM & $* * *$ & 61.90 & 75.04 & 66.17 & 67.76 & 84.05 & 70.93 \\
\hline & & 85 & 82.78 & 39.98 & 53.63 & 57.53 & 39.85 & 53.80 \\
\hline & BSVM & 90 & 88.24 & 30.51 & 49.23 & 51.89 & 37.89 & 53.01 \\
\hline & & 95 & 93.28 & 18.28 & 42.45 & 41.29 & 35.42 & 51.34 \\
\hline \multirow{5}{*}{ Glass (1) } & SVM & *** & 67.91 & 77.75 & 74.33 & 72.66 & 59.74 & 63.56 \\
\hline & CS-SVM & $* * *$ & 81.90 & 65.47 & 70.84 & 72.93 & 53.92 & 64.75 \\
\hline & & 85 & 80.40 & 68.40 & 72.28 & 74.16 & 55.29 & 65.52 \\
\hline & BSVM & 90 & 86.53 & 63.63 & 71.06 & 74.20 & 53.63 & 66.22 \\
\hline & & 95 & 92.42 & 58.18 & 69.34 & 73.33 & 51.79 & 66.38 \\
\hline \multirow[t]{5}{*}{ Thyroid (3) } & SVM & $* * *$ & 88.98 & 99.37 & 97.90 & 94.03 & 95.82 & 92.27 \\
\hline & CS-SVM & $* * *$ & 93.33 & 98.37 & 95.88 & 95.01 & 90.14 & 90.10 \\
\hline & & 85 & 93.70 & 98.64 & 97.89 & 96.14 & 91.78 & 92.73 \\
\hline & BSVM & 90 & 94.25 & 98.93 & 98.29 & 96.56 & 93.46 & 93.85 \\
\hline & & 95 & 95.23 & 98.11 & 97.69 & 96.66 & 89.10 & 92.06 \\
\hline \multirow[t]{5}{*}{ Ecoli (6) } & SVM & *** & 81.73 & 991.42 & 89.76 & 86.44 & 63.97 & 71.77 \\
\hline & CS-SVM & $* * *$ & 91.61 & 87.35 & 88.01 & 89.39 & 57.70 & 70.53 \\
\hline & & 85 & 82.63 & 91.80 & 90.29 & 87.09 & 65.26 & 72.92 \\
\hline & BSVM & 90 & 86.28 & 88.58 & 88.36 & 87.42 & 58.47 & 69.71 \\
\hline & & 95 & 94.49 & 64.90 & 69.59 & 78.31 & 33.41 & 49.37 \\
\hline \multirow[t]{5}{*}{ Bupa (1) } & SVM & *** & 45.46 & 83.81 & 67.70 & 61.73 & 67.06 & 54.19 \\
\hline & CS-SVM & $* * *$ & 65.01 & 66.08 & 65.63 & 65.31 & 58.38 & 61.31 \\
\hline & & 85 & 83.15 & 36.53 & 56.05 & 55.11 & 48.71 & 61.43 \\
\hline & BSVM & 90 & 87.59 & 26.11 & 52.02 & 47.82 & 46.22 & 60.51 \\
\hline & & 95 & 92.93 & 16.24 & 48.52 & 38.85 & 44.58 & 60.25 \\
\hline \multirow[t]{5}{*}{ Japanese (1) } & $\overline{\text { SVM }}$ & **** & "44.37 & "68.71 & "60.94 & "55.21 & "67.29 & 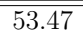 \\
\hline & CS-SVM & $* * *$ & 54.33 & 58.70 & 57.34 & 56.25 & 37.27 & 44.10 \\
\hline & & 85 & 80.59 & 28.24 & 44.49 & 47.70 & 61.97 & 70.07 \\
\hline & BSVM & 90 & 86.00 & 20.44 & 40.63 & 41.86 & 61.07 & 71.42 \\
\hline & & 95 & 91.78 & 11.93 & 36.67 & 32.83 & 60.19 & 72.71 \\
\hline \multirow[t]{5}{*}{ Australian (1) } & $\overline{\text { SVM }}$ & *** & 82.90 & 87.12 & 85.30 & 84.98 & 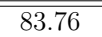 & 83.33 \\
\hline & CS-SVM & *** & 92.42 & 79.91 & 85.48 & 85.92 & 78.74 & 85.00 \\
\hline & & 85 & 81.71 & 88.91 & 85.71 & 85.23 & 85.52 & 83.57 \\
\hline & BSVM & 90 & 87.43 & 84.62 & 85.87 & 86.01 & 82.00 & 84.63 \\
\hline & & 95 & 94.26 & 70.49 & 81.07 & 81.51 & 71.91 & 81.58 \\
\hline \multirow[t]{5}{*}{ 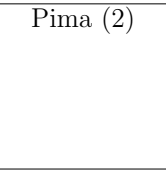 } & SVM & $* * *$ & 54.13 & 84.83 & 73.93 & 67.76 & 65.67 & 59.34 \\
\hline & CS-SVM & $* * *$ & 70.65 & 77.78 & 75.29 & 74.04 & 63.16 & 66.58 \\
\hline & & 85 & 83.93 & 63.37 & 70.60 & 72.93 & 55.12 & 66.54 \\
\hline & BSVM & 90 & 89.35 & 55.55 & 67.32 & 70.45 & 51.86 & 65.63 \\
\hline & & 95 & 94.12 & 43.47 & 61.13 & 63.96 & 47.16 & 62.83 \\
\hline \multirow[t]{5}{*}{ "German (2) } & $\overline{\text { SVM }}$ & **** & 74.87 & 64.93 & (67.86 & "69.73 & 477.78 & 58.33 \\
\hline & CS-SVM & $* * *$ & 71.96 & 71.86 & 71.89 & 71.86 & 52.36 & 60.56 \\
\hline & & 85 & 82.22 & 58.06 & 65.28 & 69.09 & 45.65 & 58.37 \\
\hline & BSVM & 90 & 87.75 & 48.34 & 60.16 & 65.13 & 42.13 & 56.92 \\
\hline & & 95 & 93.46 & 34.37 & 52.11 & 56.68 & 37.90 & 53.93 \\
\hline \multirow[t]{5}{*}{ Bank (1) } & SVM & $* * *$ & 42.43 & 90.87 & 85.25 & 62.09 & 84.01 & 56.38 \\
\hline & CS-SVM & $* * *$ & 66.92 & 82.97 & 81.12 & 74.49 & 33.89 & 44.97 \\
\hline & & 85 & 84.50 & 66.56 & 68.63 & 75.00 & 74.07 & 78.94 \\
\hline & BSVM & 90 & 89.25 & 57.53 & 61.19 & 71.66 & 70.37 & 78.69 \\
\hline & & 95 & 94.42 & 40.87 & 47.05 & 62.13 & 64.35 & 76.54 \\
\hline
\end{tabular}


- If the recall value is low for the initial SVM, then the 'price to pay' in order to improve this metric in the BSVM is a significant reduction in the specificity. This reduction can be observed in the Tae, Bupa, Japanese, Pima, and Bank datasets. Nevertheless, this does not constitute a drawback since BSVM is specifically designed for problems in which this balancce is not a critical point.

- The values for recall using BSVM are always greater than those using standard SVM, for all datasets except in the Australian dataset for BSVM with $r=0.85$. In this case, it can be seen that the value of specificity is greater with BSVM than SVM.

- It is clear that cost-sensitive SVM is better than SVM for the increase in the recall metric (except for the Iris and German datasets). Nevertheless, the value for recall in certain datasets (Iris, Tae, Bupa, Japanese, Pima, German, and Bank) can be considered poor in comparison with BSVM.

- The best result for g-mean, precision and f-value metrics in each dataset is indicated in bold in Table 3. As expected, BSVM produces the optimal hypothesis in terms of recall without penalizing too much other criteria, so that there is no model which is the best with respect to all metrics. Hence, when the objective is the recall metric, then BSVM with a high value of $r$ is the best option in all cases.

Let us now turn our attention to the case where the priority between classes is changed. Thus, in order to observe how priority affects metrics, a similar experiment to that reported previously is carried out with the Iris, Tae, Glass, Japanese and German datasets ${ }^{5}$. The results can be observed in Table 4, where symbol "*" denotes that the considered class is a non-priority class and the rest of the classes provide the priority class.

An initial observation, the priority class corresponds to the class with the highest number of examples, that is, the experiment presented is not specially addressed to manage imbalanced datasets. A number of conclusions can be drawn:

\footnotetext{
${ }^{5}$ These datasets are chosen since their specificity values in Table 3 are the lowest for the standard SVM.
} 
Table 4: Average figures of the results of the experiment for $r=0.85,0.90$, and 0.95 with a linear kernel for the Iris, Tae, Glass, Japanese, and German datasets by changing the priority between classes.

\begin{tabular}{|c|c|c|c|c|c|c|c|c|}
\hline datasets & approach & $100 r$ & recall & pecificit & accuracy & g-mean & precision & f-value \\
\hline \multirow[t]{5}{*}{ Iris $(2)^{*}$} & SVM & $* * *$ & 66.33 & 79.36 & 72.85 & 72.55 & 86.54 & 75.10 \\
\hline & CS-SVM & $* * *$ & 80.85 & 68.81 & 72.76 & 74.07 & 88.17 & 76.88 \\
\hline & & 85 & 82.70 & 55.76 & 69.23 & 67.91 & 78.90 & 80.75 \\
\hline & BSVM & 90 & 88.28 & 41.26 & 64.77 & 60.35 & 75.04 & 81.12 \\
\hline & & 95 & 93.25 & 25.62 & 59.44 & 48.88 & 71.49 & 80.93 \\
\hline \multirow{5}{*}{ Tae $(1)^{*}$} & $\overline{\text { SVM }}$ & "*** & 78.00 & 52.49 & $\bar{~} 65.25$ & $\mid 63.99$ & 83.24 & 264.38 \\
\hline & CS-SVM & *** & 75.42 & 61.68 & 66.25 & 67.89 & 84.29 & 70.96 \\
\hline & & 85 & 83.42 & 45.17 & 64.30 & 61.38 & 76.00 & 79.54 \\
\hline & BSVM & 90 & 88.36 & 34.40 & 61.38 & 55.13 & 73.71 & 80.37 \\
\hline & & 95 & 93.25 & 21.53 & 57.39 & 44.81 & 71.21 & 80.75 \\
\hline \multirow[t]{5}{*}{ Glass $(1)^{*}$} & SVM & $* * *$ & 77.76 & 67.90 & 72.83 & 72.66 & 86.26 & 75.99 \\
\hline & CS-SVM & *** & 65.32 & 81.67 & 70.66 & 72.74 & 88.30 & 74.79 \\
\hline & & 85 & 81.49 & 65.14 & 73.32 & 72.86 & 82.78 & 82.13 \\
\hline & BSVM & 90 & 86.54 & 50.77 & 68.66 & 66.28 & 78.34 & 82.23 \\
\hline & & 95 & 92.30 & 29.06 & 60.68 & 51.79 & 72.80 & 81.40 \\
\hline \multirow[t]{5}{*}{ Japanese $(2)^{*}$} & $\overline{\text { SVM }}$ & $* * *$ & 68.17 & 445.19 & 60.76 & 55.51 & 64.35 & 266.20 \\
\hline & CS-SVM & *** & 58.48 & 54.50 & 57.25 & 56.26 & 74.02 & 65.34 \\
\hline & & 85 & 82.53 & 30.26 & 66.28 & 49.97 & 63.20 & 71.58 \\
\hline & BSVM & 90 & 88.27 & 20.39 & 67.19 & 42.43 & 61.67 & 72.61 \\
\hline & & 95 & 93.86 & 10.34 & 67.90 & 31.15 & 60.30 & 73.43 \\
\hline \multirow[t]{5}{*}{ German $(2)^{*}$} & SVM & $* * *$ & 67.72 & 71.67 & 69.00 & 69.79 & 50.60 & 57.92 \\
\hline & CS-SVM & $* * *$ & 71.87 & 72.01 & 71.81 & 71.88 & 85.73 & 78.15 \\
\hline & & 85 & 82.76 & 58.96 & 75.62 & 69.85 & 46.36 & 59.43 \\
\hline & BSVM & 90 & 88.23 & 48.58 & 76.32 & 65.47 & 42.37 & 57.25 \\
\hline & & 95 & 93.84 & 31.01 & 74.98 & 53.95 & 36.83 & 52.90 \\
\hline
\end{tabular}

- Results obtained with standard SVM and cost-sensitive SVM differ from those in Table 3 since the training sets provided by the crossvalidation procedure are different. However, the performance values are very similar, with no significant differences between them, that is, the change of priority between classes exerts no noticeable effect.

- Values for specificity when using BSVM are smaller than or equal to those provided in Table 3 (except for the Japanese dataset) since the majority class is now the positive class.

- In certain cases (Glass, Japanese, and German datasets), accuracy using BSVM is better than for standard SVM because the size of the region in the feature space $\mathcal{H}$ has been increased for the majority class 
(see Section 3) and therefore the number of successes increases.

- With respect to the g-mean, precision and f-value metrics, results are qualitatively similar to those depicted in Table 3.

Finally, a third experiment is carried out on SVM classifiers using the rbf kernel with $\sigma$ (the width of $\mathrm{rbf}$ ) and $C$ being explored on a two-dimensional grid: $\sigma=\left[2^{-4}, 2^{-3}, \ldots, 2^{3}, 2^{4}\right]$ and $C=\left[2^{-4}, 2^{-3}, \ldots, 2^{9}, 2^{10}\right]$.

The criterion employed for the estimation of the generalized accuracy in the standard SVM and the cost-sensitive SVM, and the proximity ${ }^{6}$ between the recall and the threshold $r$ in the BSVM is that of the three-fold $\left(N_{\text {fold }}=3\right)$ cross-validation on the whole set of training data. This procedure is repeated 100 times in order to ensure good statistical behaviour.

The results obtained, given as percentages, are displayed in Table 5. Certain conclusions can be drawn from this empirical experimentation:

- As it is well-known, the accuracy with an rbf kernel is usually better than that with a linear kernel in standard and cost-sensitive SVM. With respect to the recall metric, sometimes it is better with a linear kernel (Tae, Japanese, German, and Bank datasets). With respect to the specificity metric, except for German datasets, the rbf SVM is better than the linear SVM.

- It can be seen that, with the Iris, Thyroid Ecoli and Australian datasets, the value of the recall metric is high for all the approaches considered. Nevertheless, BSVM is currently improving the other approaches for the Japanese and Bank datasets.

- It can be observed that using the rbf kernel, the g-mean, precision and f-value performance metrics for standard SVM have improved with respect to the linear kernel.

- With respect to the BSVM, it can be seen that the same conclusions are obtained with the rbf kernel and with the linear kernel.

\footnotetext{
${ }^{6}$ This criterion has been used since it is well-known that the rbf kernel in certain cases leads to overfitting on the training set.
} 
Table 5: Average figures of the results of the experiment with an rbf kernel.

\begin{tabular}{|c|c|c|c|c|c|c|c|c|}
\hline datasets & approach & $100 r$ & recall & ecificity & accuracy & g-mean & precision & f-value \\
\hline \multirow[t]{5}{*}{ Iris (1) } & SVM & $* * *$ & 95.20 & 96.01 & 95.69 & 95.60 & 92.27 & 93.71 \\
\hline & CS-SVM & $* * *$ & 96.06 & 96.37 & 96.27 & 96.15 & 93.40 & 94.51 \\
\hline & & 85 & 85.29 & 97.44 & 93.35 & 91.16 & 94.34 & 89.59 \\
\hline & BSVM & 90 & 89.48 & 97.69 & 94.90 & 93.50 & 95.10 & 92.20 \\
\hline & & 95 & 95.08 & 87.58 & 90.03 & 91.26 & 79.29 & 86.47 \\
\hline \multirow[t]{5}{*}{ Tae (1) } & SVM & *** & 45.19 & 85.81 & 72.16 & 62.16 & 60.48 & 51.73 \\
\hline & CS-SVM & $* * *$ & 38.62 & 97.45 & 78.36 & 60.82 & 88.58 & 53.00 \\
\hline & & 85 & 79.90 & 47.49 & 57.97 & 61.60 & 42.23 & 55.25 \\
\hline & BSVM & 90 & 84.35 & 32.20 & 49.09 & 52.12 & 37.41 & 51.83 \\
\hline & & 95 & 89.28 & 22.60 & 44.17 & 44.93 & 35.66 & 50.96 \\
\hline \multirow{5}{*}{ Glass (1) } & SVM & *** & 70.69 & 90.06 & 83.60 & 79.79 & 77.56 & 73.96 \\
\hline & CS-SVM & $* * *$ & 82.01 & 75.73 & 79.97 & 78.61 & 67.71 & 71.14 \\
\hline & & 85 & 86.73 & 59.81 & 68.34 & 72.02 & 51.20 & 64.39 \\
\hline & BSVM & 90 & 89.36 & 44.82 & 59.19 & 63.28 & 44.05 & 59.01 \\
\hline & & 95 & 93.18 & 52.37 & 65.64 & 69.86 & 48.74 & 64.00 \\
\hline \multirow[t]{5}{*}{ Thyroid (3) } & SVM & *** & 89.22 & 98.89 & 97.53 & 93.93 & 92.89 & 91.02 \\
\hline & CS-SVM & $* * *$ & 91.73 & 98.75 & 97.77 & 94.96 & 93.05 & 91.77 \\
\hline & & 85 & 86.96 & 98.80 & 97.17 & 92.69 & 92.17 & 89.49 \\
\hline & BSVM & 90 & 89.67 & 98.76 & 97.53 & 94.11 & 92.17 & 90.90 \\
\hline & & 95 & 94.78 & 98.35 & 97.87 & 96.55 & 90.31 & 92.49 \\
\hline \multirow[t]{5}{*}{ Ecoli (6) } & SVM & *** & 85.76 & 97.66 & 95.75 & 91.52 & 87.23 & 86.49 \\
\hline & CS-SVM & $* * *$ & 83.31 & 96.94 & 94.80 & 89.75 & 84.06 & 83.36 \\
\hline & & 85 & 85.18 & 90.32 & 89.49 & 87.71 & 62.11 & 71.84 \\
\hline & BSVM & 90 & 89.51 & 75.67 & 77.85 & 82.30 & 40.68 & 55.94 \\
\hline & & 95 & 94.28 & 73.87 & 77.10 & 83.46 & 40.21 & 56.38 \\
\hline \multirow[t]{5}{*}{ Bupa (1) } & SVM & *** & 48.57 & 85.17 & 69.61 & 64.32 & 70.36 & 57.47 \\
\hline & CS-SVM & $* * *$ & 67.17 & 74.06 & 71.17 & 70.39 & 65.47 & 66.14 \\
\hline & & 85 & 83.88 & 28.92 & 51.93 & 49.25 & 46.11 & 59.50 \\
\hline & BSVM & 90 & 87.30 & 35.07 & 56.98 & 55.33 & 49.36 & 63.07 \\
\hline & & 95 & 96.75 & 7.88 & 45.26 & 27.61 & 43.23 & 59.76 \\
\hline \multirow[t]{5}{*}{ Japanese (1) } & $\overline{\mathrm{SVM}}$ & **** & "02.56 & "98.04 & "68.34 & 15.85 & ב37.14 & (04.80 \\
\hline & CS-SVM & $* * *$ & 00.33 & 98.48 & 67.95 & 02.26 & 07.73 & 00.55 \\
\hline & & 85 & 72.55 & 27.04 & 41.11 & 44.29 & 30.97 & 43.41 \\
\hline & BSVM & 90 & 76.77 & 29.31 & 44.00 & 47.44 & 32.88 & 46.04 \\
\hline & & 95 & 84.79 & 19.98 & 40.09 & 41.16 & 32.34 & 46.82 \\
\hline \multirow[t]{5}{*}{ Australian (1) } & SVM & **** & 92.26 & 79.81 & 85.33 & 85.81 & 78.55 & 84.86 \\
\hline & CS-SVM & $* * *$ & 92.45 & 80.55 & 85.84 & 86.27 & 79.28 & 85.33 \\
\hline & & 85 & 81.13 & 89.39 & 85.72 & 85.16 & 85.97 & 83.48 \\
\hline & BSVM & 90 & 88.29 & 84.58 & 86.24 & 86.41 & 82.11 & 85.08 \\
\hline & & 95 & 94.27 & 72.70 & 82.31 & 82.79 & 73.46 & 82.58 \\
\hline \multirow[t]{5}{*}{ Pima (2) } & SVM & *** & 61.22 & 81.57 & 74.45 & 70.67 & 64.03 & 62.60 \\
\hline & CS-SVM & $* * *$ & 68.94 & 76.83 & 74.08 & 72.67 & 61.59 & 64.92 \\
\hline & & 85 & 83.56 & 63.18 & 70.31 & 72.66 & 54.88 & 66.25 \\
\hline & BSVM & 90 & 88.90 & 56.24 & 67.65 & 70.71 & 52.13 & 65.72 \\
\hline & & 95 & 94.12 & 45.70 & 62.60 & 65.58 & 48.16 & 63.72 \\
\hline \multirow[t]{5}{*}{ "German (2) } & SVM & **** & 28.55 & 93.05 & 73.66 & 51.55 & 63.77 & 39.45 \\
\hline & CS-SVM & $* * *$ & 65.90 & 75.03 & 72.29 & 70.25 & 53.20 & 58.79 \\
\hline & & 85 & 85.14 & 40.68 & 54.03 & 58.85 & 38.09 & 52.63 \\
\hline & BSVM & 90 & 89.89 & 33.40 & 50.32 & 54.79 & 36.65 & 52.07 \\
\hline & & 95 & 96.48 & 07.49 & 34.19 & 26.88 & 30.89 & 46.80 \\
\hline \multirow[t]{5}{*}{ Bank (1) } & SVM & $* * *$ & 00.66 & 99.61 & 88.21 & 08.14 & 18.07 & 01.28 \\
\hline & CS-SVM & $* * *$ & 00.23 & 99.68 & 88.22 & 03.71 & 11.95 & 00.45 \\
\hline & & 85 & 79.27 & 54.77 & 57.61 & 65.89 & 18.58 & 30.11 \\
\hline & BSVM & 90 & 87.07 & 43.30 & 48.32 & 61.40 & 16.67 & 27.98 \\
\hline & & 95 & 93.40 & 25.61 & 33.42 & 48.91 & 14.06 & 24.43 \\
\hline
\end{tabular}




\section{Conclusions}

A post-processing technique for Support Vector Machines, called BSVM, has been introduced to deal with datasets where one class, the positive class, is considered more relevant than another class in binary classification prob-

lems. Hence, BSVM is designed for the case when it is non-critical to increase the true positive ratio in exchange for an increase in the false positive rate.

By modifying the bias on the standard SVM, the aim of BSVM is, given a fixed minimum value for recall, to maximize specificity on the training set.

Empirical results show that due to the generalization capacity of SVMs, recall on the test set in the BSVM is close to the fixed level $r$, that is, BSVM produces a good hypothesis in terms of recall.

Furthermore, the algorithm presented can easily be applied in practice in order to address to real-world complexities, such as those faced by practitioners with an interest in benefitting management.

\section{Acknowledgements}

This research is partially supported by the projects of the Spanish Ministry of Economy and Competitiveness HERMES (TIN2013-46801-C4-1-R), PATRICIA (TIN2012-38416-C03-01) and Simon (TIC-8052) of the Andalusian Regional Ministry of Economy, Innovation and Science. Haydemar Nuñez acknowledges the I3 grant has been partly supported by the PG-037678-2009/1 project from the Central University of Venezuela. Yenny Leal was supported by the Agency for Management of University and Research Grants of the Government of Catalonia, Spain (Beatriu de Pinós [BP-DGR2013]).

\section{References}

[1] C. Angulo, F. Ruiz, L. González, and J.A. Ortega. Multi-classification by using tri-class SVM. Neural Proceeding Letters, 23(1):89-101, 2006.

[2] C.L. Blake and C.J. Merz. UCI repository of machine learning databases, 1998.

[3] G. Casella and R. Berger. Statistical Inference. Duxbury Resource Center, 2001. 
[4] G. Cohen, M. Hilario, H. Sax, S. Hugonnet, and A. Geissbuhler. Learning from imbalanced data in surveillance of nosocomial infection. Artif. Intell. Med., 37:7-18, May 2006.

[5] N. Cristianini and J. Shawe-Taylor. An introduction to Support Vector Machines and other kernel-based learning methods. Cambridge University press 2000, 2000.

[6] R.S. Debreceny and G.L. Gray. Data mining journal entries for fraud detection: An exploratory study. International Journal of Accounting Information Systems, 11(3):157 - 181, 2010.

[7] C. Elkan. The foundations of cost-sensitive learning. In Proceedings of the 17th International Joint Conference on Artificial Intelligence Volume 2, IJCAI'01, pages 973-978. Morgan Kaufmann Publishers Inc., 2001.

[8] T. Fawcett. An introduction to ROC analysis. Pattern Recogn. Lett., 27:861-874, 2006.

[9] L. González, C. Angulo, F. Velasco, and A. Català. Unified dual for bi-class SVM approaches. Pattern Recognition, 38(10):1772-1774, 2005.

[10] L. González, C. Angulo, F. Velasco, and A. Català. Dual unification of bi-class support vector machine formulations. Pattern Recognition, 39(7):1325-1332, 2006.

[11] L. Gonzalez-Abril, C. Angulo, F. Velasco, and J.A. Ortega. A note on the bias in SVMs for multiclassification. IEEE Trans. Neural Netw., 19(4):723-725, 2008.

[12] L. Gonzalez-Abril, H. Nuñez, C. Angulo, and F. Velasco. GSVM: An SVM for handling imbalanced accuracy between classes in biclassification problems. Applied Soft Computing, 17:23 - 31, 2014.

[13] L. Gonzalez-Abril, F. Velasco, J.A. Ortega, and L. Franco. Support vector machines for classification of input vectors with different metrics. Computers \& Mathematics with Applications, 61(9):2874 - 2878, 2011.

[14] H. He and E.A. Garcia. Learning from imbalanced data. IEEE Trans. Know. and Data Eng., 21(9):1263-1284, September 2009. 
[15] C-W. Hsu and C-J. Lin. A comparison of methods for multiclass support vector machine. IEEE Transactions on Neural Networks, 13(2):415-425, March 2002.

[16] M. Karabatak and M. Cevdet Ince. An expert system for detection of breast cancer based on association rules and neural network. Expert Systems with Applications, 36(2, Part 2):3465 - 3469, 2009.

[17] B. Krawczyk. Learning from imbalanced data: open challenges and future directions. Progress in Artificial Intelligence, 5(4):221-232, 2016.

[18] W.Y. Lin, Y.H. Hu, and C.F. Tsai. Machine learning in financial crisis prediction: A survey. IEEE Transactions on Systems, Man, and Cybernetics, Part C (Applications and Reviews), 42(4):421-436, 2012.

[19] V. López, A. Fernández, S. García, V. Palade, and F. Herrera. An insight into classification with imbalanced data: Empirical results and current trends on using data intrinsic characteristics. Information Sciences, 250:113 - 141, 2013.

[20] S. Moro, P. Cortez, and P. Rita. A data-driven approach to predict the success of bank telemarketing. Decision Support Systems, 62:22 - 31, 2014.

[21] D.L. Olson, D. Delen, and Y. Meng. Comparative analysis of data mining models for bankruptcy prediction. Decision Support Systems, 52(2):464-473, 2012.

[22] L. Oneto, S. Ridella, and D. Anguita. Tikhonov, Ivanov and Morozov regularization for support vector machine learning. Machine Learning, 103(1):103-136, 2016.

[23] R.B. Rao, S. Krishnan, and R.S. Niculescu. Data mining for improved cardiac care. ACM SIGKDD Explorations Newsletter, 8(1):3-10, 2006.

[24] P. Ravisankar, V. Ravi, G. Raghava Rao, and I. Bose. Detection of financial statement fraud and feature selection using data mining techniques. Decision Support Systems, 50(2):491 - 500, 2011.

[25] V. Vapnik. Statistical Learning Theory. John Wiley \& Sons, New York, 1998. 
[26] V. Vapnik. The Nature of Statistical Learning Theory (Information Science and Statistics). Springer, November 1999.

[27] Z. Yang, W. You, and G. Ji. Using partial least squares and support vector machines for bankruptcy prediction. Expert Systems with Applications, 38(7):8336 - 8342, 2011.

[28] J.S. Yazdi, F. Kalantary, and H.S. Yazdi. Prediction of liquefaction potential based on cpt up-sampling. Computers \& Geosciences, 44:10$23,2012$.

[29] J.S. Yoon and Y.S. Kwon. A practical approach to bankruptcy prediction for small businesses: Substituting the unavailable financial data for credit card sales information. Expert Systems with Applications, 37(5):3624 - 3629, 2010.

[30] M. Zieba, J.M. Tomczak, M. Lubicz, and J. Swiatek. Boosted SVM for extracting rules from imbalanced data in application to prediction of the post-operative life expectancy in the lung cancer patients. Applied Soft Computing, 14, Part A:99 - 108, 2014. 
LaTeX Source Files
Click here to download LaTeX Source Files: ASOC-D-16-02638_Revised_Second.tex

LaTeX Source Files
Click here to download LaTeX Source Files: ASOC-D-16-02638_Revised_Second.tex Click here to download LaTeX Source Files: AsOC-D-16-02638_Revised_Secondtex

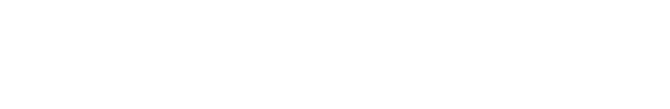

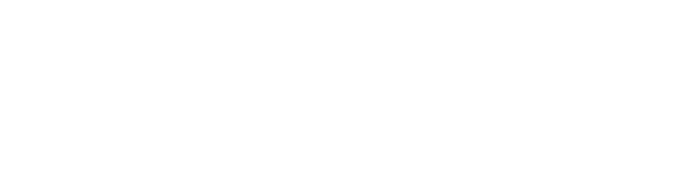
(1) (1) (1) (1) (1) (1) . . . . . . . . 
- An SVM's post-processing strategy is introduced which is specially designed to handle bi-classication problems where one class (positive) is considered of greater significance than the other (negative).

- Maximizing the true positive rate (Recall) is of a higher priority than improving the accuracy of the overall classifier.

- A post-processing technique is provided, such that, given a fixed value for Recall, the true negative rate (Specificity) is maximized. 


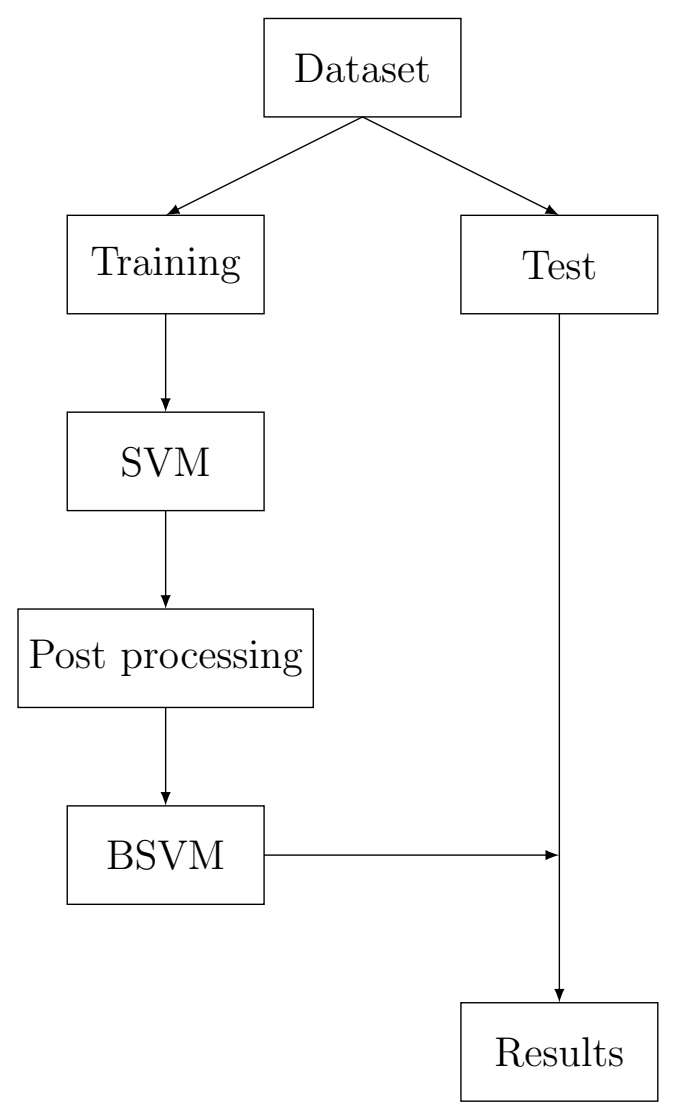


DATE: $06^{\text {th }}$ April, 2017

LESLEY JOAN BURRDGE

NE X-5971058-M

Cl Guadalbullón, $15,11 \mathrm{C}$

41013 SEVILA

\section{To whom it may concern:}

I have proofread and corrected the English of the paper:

"A support vector machine for handling binary

classification problems with a priority class",

by

Luis Gonzalez-Abril, Cecilio Angulo,

Haydenar Nuñez and Yenny Leal.

Yours faithfilly,

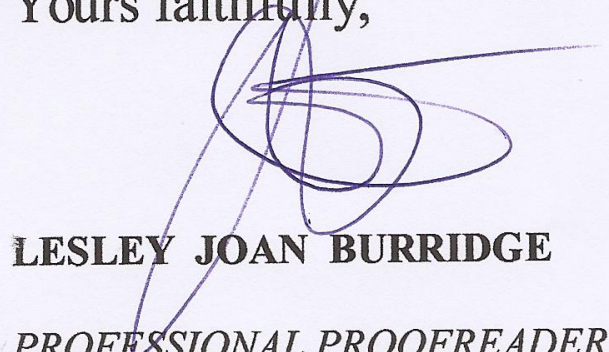

PROFESSIONAL PROOFREADER AND TRANSLATOR 